m).Of the 29 lupus patients, 13 charts were retrieved. The mean age at diagnosis was 13.3 years(SD 3.4) from 6-18 years old. Oedema(53.8\%), fever(46.1\%), abdominal pain (38.4\%) and easy fatigability (30.7\%) were the most common features at disease onset while renal involvement (84.6\%), malar rash $(53.8 \%)$ and oral ulceration(46.1\%) were common at the time of diagnosis. All of 9 patients with ANA titers were positive. Anti-dsDNA antibodies were high in 3 patients. Low complement values were seen in $83.3 \%$. The follow-up period ranged from $0.2-2 y$ with a mean duration of $1.2 \pm 0.6$ y.Four went into remission but 3 patients died, 3 patients transitioned to adult section and 3 were lost to follow-up.

Conclusions Oedema and renal involvement were the most common feature at the onset and at the the time of the disease, respectively. All male lupus patients had positive ANA and low C3 results. Causes of death were:active disease, sepsis and DIC. Early recognition and diagnosis will lead to prompt institution of treatment that will benefit lupus patients.

\section{ATOPY IN CHILDREN WITH JUVENILE SYSTEMIC LUPUS ERYTHEMATOSUS IS ASSOCIATED WITH SEVERE DISEASE}

${ }^{1} \mathrm{G}$ Ruru*, 'L Liangjing, ${ }^{2} \mathrm{C}$ Lanfang, ${ }^{2} \mathrm{C}$ Junjia, ${ }^{2} \mathrm{Z}$ Yanqing. ${ }^{1}$ Renji Hospital-School of Medicine-Shanghai Jiao Tong University, Rheumatology, Shanghai, China; ${ }^{2}$ Renji HospitalSchool of Medicine-Shanghai Jiao Tong University, Pediactirc, Shanghai, China

\subsection{6/lupus-2017-000215.140}

Background and Aims We aimed to assess the influence of coexisting atopy on the prognosis of juvenile systemic lupus erythematosus (JSLE)

Methods Patients diagnosed with JSLE between October 2005 and April 2016 were enrolled in a prospective cohort study and followed for 2 years. Management of patients was evaluated using SLEDAI-2K score. Eighty JSLE patients were enrolled at diagnosis and were divided into those with atopy and those without.

Results Atopic patients had significantly higher SLEDAI-2K at disease onset (16.09 vs. 11.18), higher anti-double-stranded DNA (66.58 vs. $44.55 \mathrm{IU} / \mathrm{ml}$ ), higher erythrocyte sedimentation rate $(52.89$ vs. $38.27 \mathrm{~mm} / \mathrm{h})$, higher percentage of total B-cells (25.85 vs. 19.51\%), lower percentage (7.26 vs. 9.03\%) and activity $(9.92$ vs. $11.32 \%)$ of natural killer cells, lower complement C3 (0.51 vs. $0.69 \mathrm{~g} / \mathrm{L})$, and lower complement C4 (0.06 vs. $0.12 \mathrm{~g} / \mathrm{L}) \quad(\mathrm{p}<0.05$ for all comparisons). At 1 month, $3,6,12,18$ and 24 months, JSLE patients with atopy reached higher SLEDAI-2K and lower $\triangle$ SLEDAI-2K improvement rate (at 1 month, 8.34 vs. 4.71 and 43.63 vs. $57.95 \%$, respectively; at 3 months, 8.57 vs. 2.62 and 48.39 vs. $75.10 \%$, respectively; at 6 months, 6.91 vs. 2.38 and 53.59 vs. $77.26 \%$, respectively; at 12 months, 4.71 vs. 1.80 and 69.54 vs. $84.10 \%$, respectively; at 18 months, 4.66 vs. 2.02 and 68.14 vs. $82.93 \%$, respectively; at 24 months, 8.57 vs. 2.62 and 70.00 vs. $81.88 \%$, respectively; all $\mathrm{p}<0.05$ ).

Conclusions Co-existing atopy in children with JSLE may exert an adverse influence on JSLE, with atopic patients manifesting more severe disease at diagnosis and poorer outcome.

\section{SOLUBLE RECEPTOR ACTIVATOR OF NUCLEAR FACTOR $K$ B LIGAND (S RANK-L) LEVELS IN PAEDIATRIC ONSET SLE}

S Sandal*, A Rawat, A Gupta, S Singh. PGIMER, Paediatrics, Chandigarh, India

\subsection{6/lupus-2017-000215.141}

Background and aims Receptor Activator of Nuclear Factor $\kappa$ B (RANK), its ligand (RANKL) and osteoprotegerin are the key mediators of bone remodelling and the final effector pathway in osteoclast development and differentiation. The data on RANKL axis in paediatric Systemic Lupus Erythematosus (SLE) is lacking. Thus we proposed to estimate serum sRANKL levels in paediatric SLE and to correlate sRANKL levels with the SLE disease activity

Methods Consecutive children with SLE attending Paediatric Rheumatology Clinic of Advanced Paediatrics Centre, PGIMER, Chandigarh were enrolled. The study group was divided into active (with ongoing disease activity) and inactive (no disease activity) subgroups based on SLE disease activity index (SLEDAI) scores. The sRANKL ligand levels were measured at enrollment using an enzyme- linked immunosorbent assay (sRANKL - ELISA MyBioSource@, USA).

Results Thirty-one children (12 boys) with a mean age of 13.4 \pm 3.2 years were included. The median (interquartile range) sRANKL level of the cohort was 52.3 (24.1, 66.4) $\mathrm{pg} / \mathrm{mL}$. Serum RANKL levels were not significantly different in active and inactive disease subgroups [median (interquartile range): $55.2(21.3,66.4) \mathrm{pg} / \mathrm{mL}$ versus 53.3 (29.3, 64.9) $\mathrm{pg} / \mathrm{mL}$, respectively] $(p=0.89)$. There was no statistically significant correlation between sRANKL levels and SLEDAI scores, Spearman correlation coefficient $\mathrm{rho}=0.083, \mathrm{p}=0.65$,

Conclusions There was no significant difference in sRANKL levels between the inactive and active disease group. Also there appears no correlation between sRANKL level and SLEDAI scores.

\section{CLINICAL PROFILE AND LONG TERM OUTCOME OF CHILDHOOD SYSTEMIC LUPUS ERYTHEMATOSUS}

S SAWHNEY*, A SHIVPURI, M AGARWAL. Sir Ganga Ram Hospital, Paediatric Rheumatology, NEW DELHI, India

\subsection{6/lupus-2017-000215.142}

Background and aims Systematic study of all diseases is essential to understand the spectrum of the disease presentation, the severity of the disease and the outcome. There is paucity of data from India on details of paediatric SLE.

This study aims to define:

- Describe the clinical and immunological profile of SLE within six months of disease onset in three age categories

- To compare the performance of ACR 1997 criteria vs SLICC 2012 criteria to classify disease in first 6 months of onset

- To define the mean value of SLEDAI at presentation and over a 5 year follow up

Methods Children attending the paediatric rheumatology clinic from January 2009 to September 2016 were included and details recorded. 